\title{
Using Ant Algorithm to Find the Optimal Critical Path of a Projects Network
}

Ziyad A. Mohammed

ziyadahmedmz1983@gmail.com
Sama T. Al_Obaidy

samatalee843@gmail.com

\section{College of Computer Science and Mathematics \\ University of Mosul, Mosul, Iraq}

Received on: 08/12/2020

Accepted on: 10/01/2021

\begin{abstract}
Intelligent techniques to solve the problem of decision-making in project management, apart from the methods of operations research, the choice was made on one of the algorithms of crowd intelligence represented by the Ant Colony Optimization algorithm (ACO)to solve the matter of finding the optimal critical path for the enterprise business network because the business network is more Networks tradition the behavior of the ant colony system to find the optimal critical path for the Critical Path Network(CPN) as. You own a project beginning contract (the first event) equivalent to an ant hill.The project end contract (the last event) is equivalent to the food site.The matter of finding the optimal critical path for the project is equivalent to the search process to find an optimal (the shortest) path between the nest and the food site.

The program ANTOCPN, written in Matlab language on a virtual business network. The program is featuring by its efficiency, accuracy of results, and the possibility of applying it to any business network, regard of the degree of complexity in terms of the number of paths (activities), whether real or imaginary, smoothly and easily. Also, the results of the ANTOCPN algorithm program were compared with the results of the genetic algorithm program for the same question GAOCPN for previous research, and the ant algorithm proved its worth in terms of speed in obtaining the optimal solution.
\end{abstract}

Keywords: Ant Colony Optimization (ACO), Critical Path Method (CPM), projects networks.

$$
\begin{aligned}
& \text { استخدام خوارزمية نمل لإيجاد المسار الحرج الأمثل لشبكة المشاريع }
\end{aligned}
$$

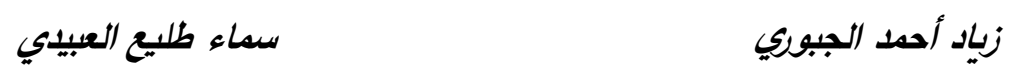

$$
\begin{aligned}
& \text { كلية علوم الحاسوب والرياضيات } \\
& \text { جامعة الدوصل، الدوصل، العراق }
\end{aligned}
$$

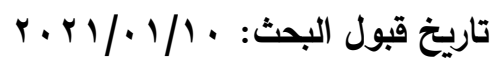

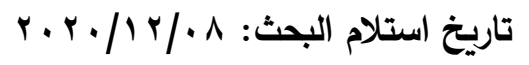

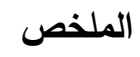


Critical Path Network (CPN) نهاية المشروع (الحدث الأخير) تعادل مصدر الطعام. مسألة إيجاد المسار الحرج الأمثل للمشروع يعادل عملية البحث لإيجاد أمثل (أقصر ) مسار بين العش وموقع الطعام. طُبق البرنامج ANTOCPN الذي كتب بلغة ماتلاب (Matlab) على شبكة أعمال افتراضية، ويتميز

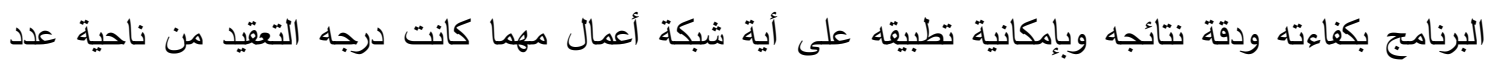

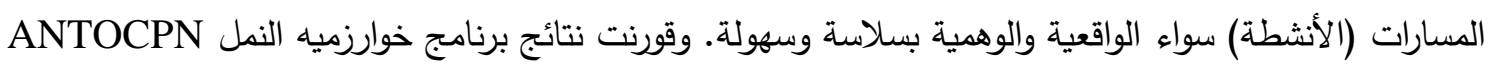

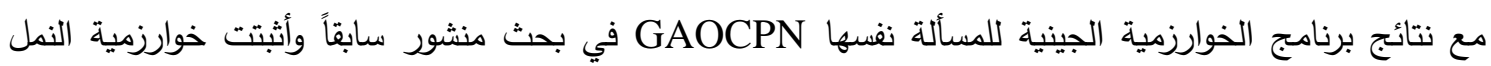
جدارتها من سرعة الحصول على الحل الأمثل. الكلمات المفتاحية: أمثلية مستعمرة النمل، المسار الحرج الأمثل، شبكات المشاريع.

1

تعدُ خوارزمية مستعمرة النمل Ant Colony Optimization (ACO) من خوارزميات البحث الحسبية

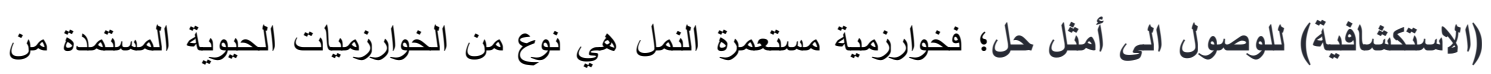

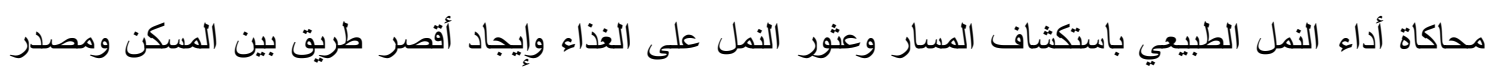
الغذاء في الطبيعة، ويمكن للنمل ترك مادة عطرية كيميائية (Pheromone) على المسار الذي يمر بهاه، وبإمكان

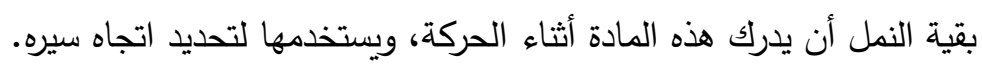

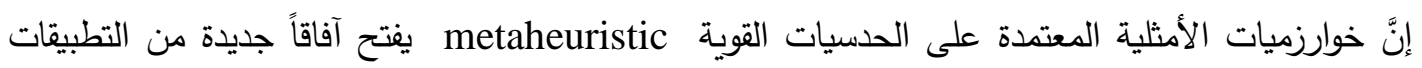
في الحياة الحقيقية، وقد أدخل العالم ماركو دوريغو نظام مستعمرة النمل في أوائل التسعينيات من القرن الماضي

وجدولة الأعمال هي عملية صياغة خطة (شبكة) تتضمن توقيت إتمام المهام لمشروع معين وفق تسلسل محدد يتيح للمؤسسة تحقيق أهدافها في وقت مثالي، وبالنسبة لمشاريع الأعمال تعد وتمثل رسومياً بثكل عقد تمثل تونل

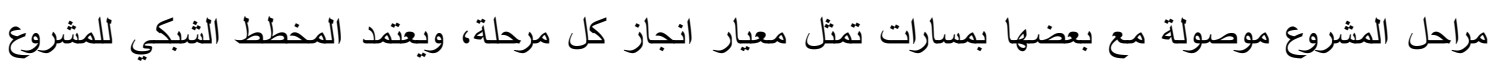
لرصد المعيار الإجمالي للمشروع ونسبة انجاز المشروع[9] واستخدمت فكرة التشثيل الرسومي لشبكة أعمال المشاريع التي ظهرت في آواخر الخمسينيات من القرن الماضي.

\section{2- نظرة عامة عن مستعمرة النمل الطبيعية}

النمل كائنات شبه عمياء وعديمة الذاكرة وذات دورة حياة قصيرة الأمد ومع ذلك تتمتع بقدرة هائلة على تثكيل شبكة أنموذجية من المسالك بين مسكنها وأماكن وجود الغذاء، وفي العالم الطبيعي يتجول النمل (مبدئيًا) بشكل عشوائي، وعند العثور على الطعام يعود إلى مستعمرتهِ بعد ترك مادة عطرية (أثر) على المسارات التي سلكها؛

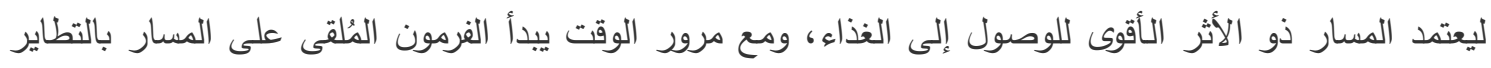

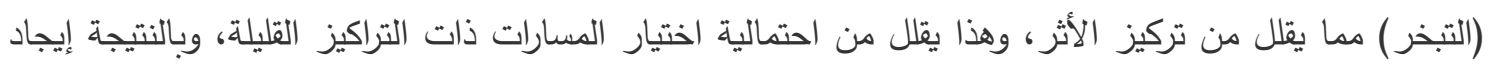
أقصر مسار بين المستعمرة ومصدر الغذاء. 
وفكرة خوارزمية مستعمرة النمل الاصطناعي هي تقليد ومحاكاة سلوك النمل بحيث يتيح الوصول إلى الحلول

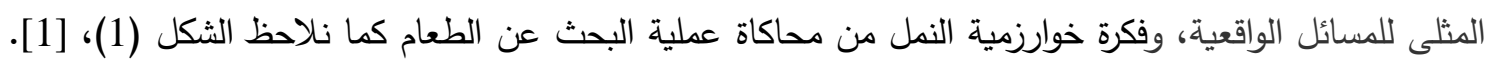
وخطوات سلوك النمل تتمثل بالآتي: 1- بدايةً مجموعة من النمل المستكثف ينطلق من المسكن في عدة اتجاهات بمسارات عشوائية للبحث عن بلاتين مصادر للغذاء.

2- تتواصل النملة مع أفراد مجموعتها بواسطة فرز مادة كيميائية عطرية Pheromone على المسار الذي تسلكه، لإرشاد المجموعة على مسارها (موقعها).

3- عندما تجد النملة مصدر الطعام تأخذ كمية منه وتسلك المسار الذي يحوي أكبر كمية فيرمون للعودة الى كئل المسكن، وأيضاً أثناء عودتها تقرز كمية منه.

4- تتحس بقية أفراد مستعمرة النمل مسار النملة التي اكتشفت المصدر الغذائي أولاً، وعليه ستزداد حركة النمل على هذا المسار، ومع زيادة تركيز المادة العطرية المفرزة عليه سيزداد انجذاب النمل إليه؛ كونه المسار المثالي (الأقصر والأسهل) بين المسكن ومصدر الغذاء. تركيز.

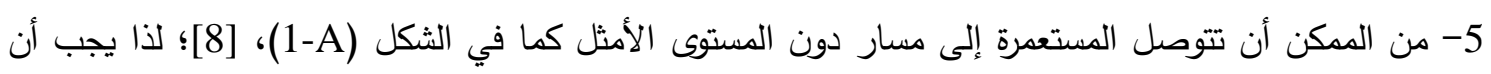
يُرسل النمل الباحث لاستكثاف المسار الأمثل (الأقصر) بين المسكن ومصدر الغذاء الغناء عن طريق جمع مسارات

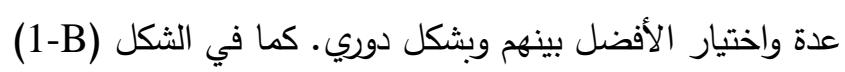

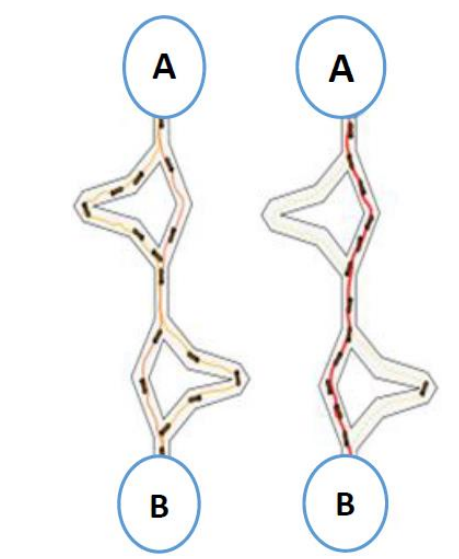

الثكل (1-A): استكثاف المسار الأمثل

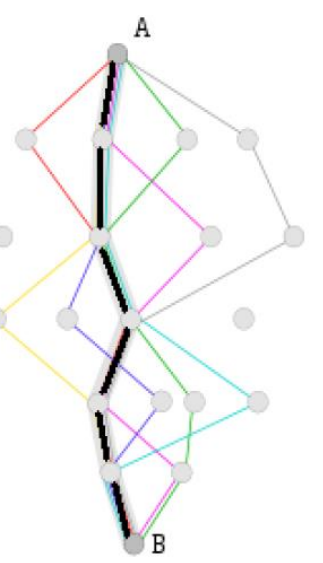

الثكل (1-B): اختيار المسار الأمثل

الثكل (1): آلية البحث لدى النمل لاستكثاف مسارات للصدر الطعام (1) (1):

3- المفاهيم الأساسية لتمثيل وإعداد المخطط الثبكي للمشروع

الثبكة هي تمثيل رسومي للمشروع؛ إذ إنَّ تحليل الثبكات يوفر طريقة عملية لرصد التقدم المحرز في انجاز

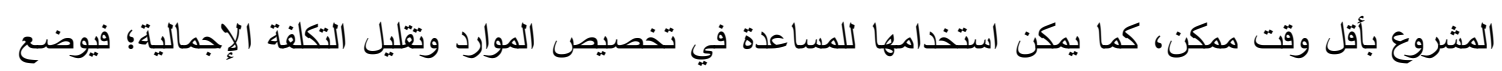
أحد المعايير (الوقت، الكلفة، الربح) على النشاط، وقد نستعين بنشاط وهمي أو أكثر . توصف شيكنة شيكة الأعمال

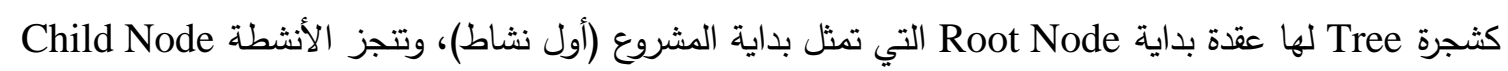

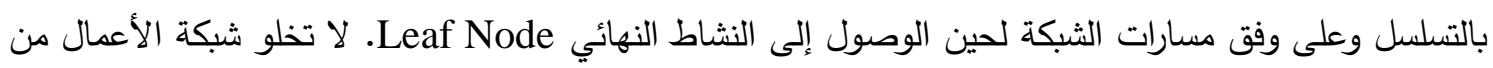
نشاط وهمي لإتمام مسار كامل وكلفة هذا المسار هي صفر ؛ إذ يرمز لبداية الثبكة بالعقدة الأولى ويستمر ترميز العقد تصاعدياً وباتجاه اليمين لغاية آخر عقدة بالثبكة، وكما موضح فئس في الثكل (2)، [2]. 


\section{قيمة النشاط}

1

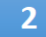

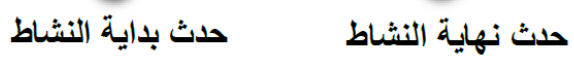
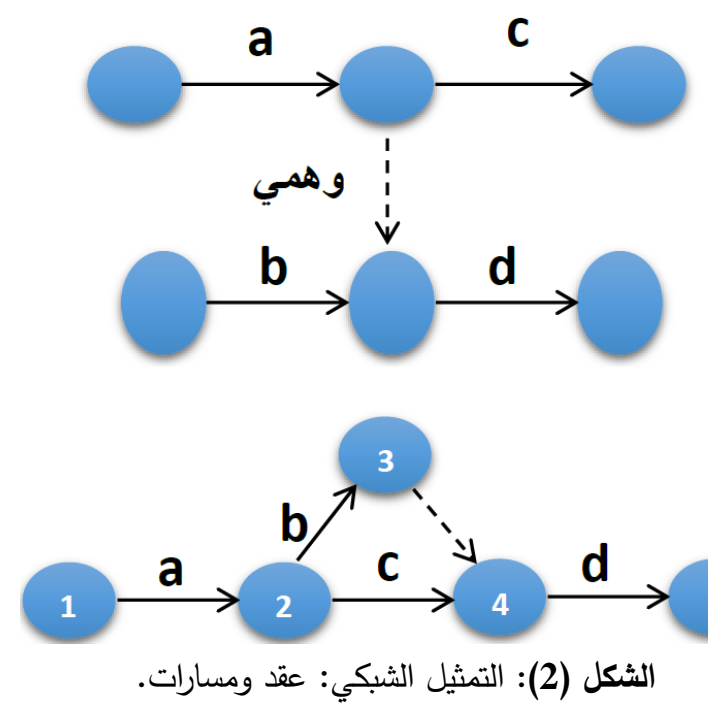

أ - النشاط Activity: مجموعة من المهام يتطلب أداؤها مجموعة من الموارد المادية: البشرية، الوقت، المواد،

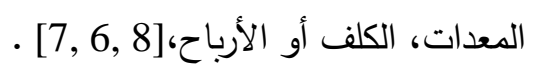

- النشاط الفعلي: هي الأنشطة التي تستغرق وقت وتستلزم موارد مادية وبشرية ويعبر عنها بخط متصل.

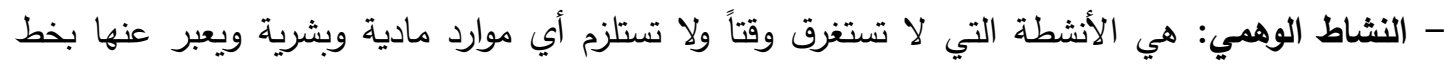

$$
\text { متقطع وتكون قيمته =0. }
$$

ب- الحدث Event: يعبر عنه بعقدة، توضع العقد بين الأنشطة لتبين نهاية نشاط وبداية نشاط آخر، والعقدة الأخيرة في الثبكة تمثل نهاية المشروع ككل.

ج- المسار Path: يمثل سلسلة من الأسهم المتعاقبة تبدأ بحدث البداية وتتنهي بحدث النهاية.

4- أسلوب المسار الحرج Critical Path Method (CPM)

يُعد أسلوب المسار الحرج (CPM) أسلوباً تقديرياً اعتمد في التخطيط والتحليل والتحكم في تقدم وإنجاز

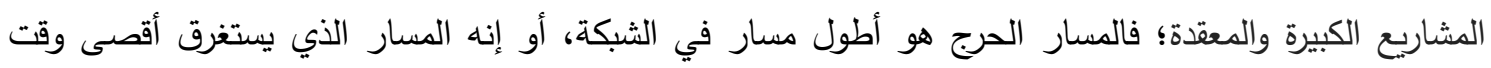

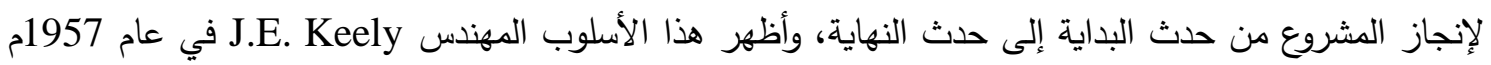
في شركة emington-Rand والمهند من W.R. Walker في شركة Dupont، [10]. وذلك لغرض جدولة عمليات التعطل بسبب الصيانة في مصنع المواد الكيميائية، وبدأت شركة فورد للسيارات وجنرال موتورز وأبل للتلفونات باستخدام أسلوب المسار الحرج، واستخدمته عام 1973 القوات المسلحة المصرية في عبور قناة السويس واستخدام لأول مرة في العراق عام 1975 في بناء سد حمرين. إن الغرض الرئيس من هذا الأسلوب التأكد التام من فن فئن

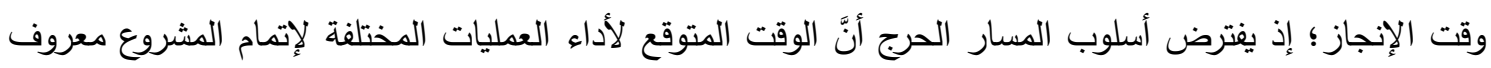
والعلاقة بين الموارد المستخدمة والوقت المطلوب لأداء العمليات المختلفة معلومة أيضاً [3]. 
5- دراسات سابقة:

تم العمل على خوارزميات عدة لتحسين مستعمرة النمل (ACO) في حل العديد من مشكلات التحسين

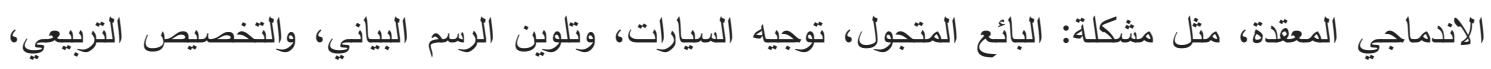
وتحسين حركة المرور، ومشكلة جدولة المتاجر وأثبت البحث قدرة نظام Ant على إنتاج مسار حرج جيد ومسارات دون الحرجة N. Ravi Shankar, P. Phani Bushan Rao, S. Siresha and K. Usha) Madhuri 2011,[11]. كذلك قام كل من (سماء طليع عزيز، نعم عبدالمنعم عبدالمجيد، لمياء جاسم محمد) بعمل بحث على

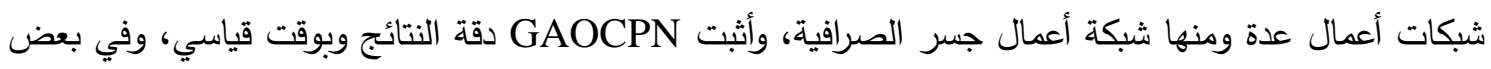
الأحيان أعطى نتائج مثلى أفضل من الطرائق التقليدية (البرمجة الديناميكية) ونتائج مطابقة للبرنامج الجاهز ونئي Win_QSB وقد قام (خالد ضاري الطائي، ابتهال هاشم رحيم) باستخدام خوارزمية مستعمرة النمل في إيجاد الحل الأمثل

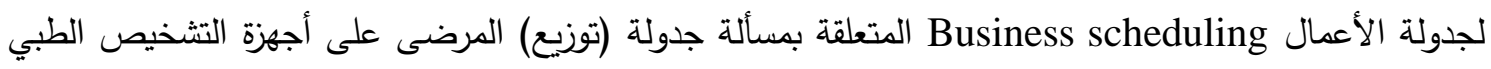
في مستشفى العلوم العصبية وبشكل يضمن إتمام تثخيصهم بوقت مثالي (أقل وقت)، وقورنت بالخوارزمية الجينية

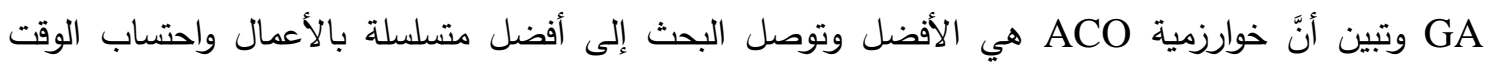

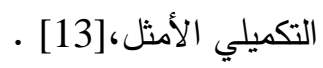

\section{6- خطوات خوارزمية مستعمرة النمل الاصطناعي (ACO)}

تمثل خوارزمية مستعمرة النمل الذكائية محاكاة لسلوك النمل في الطبيعة؛ إذ إن كل نملة تبني حلاً للمسألة

المطروحة : [4,3

\section{اولاً/ تهيئة معاملات خوارزمية ACOA}

1 الحدسية على التوالي ويصفان مدى قوة جذب المادة العطرية للنمل.

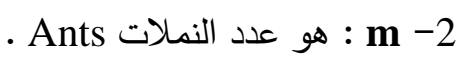

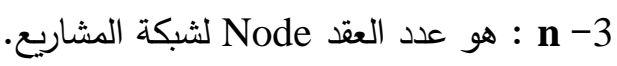

tour -4 : معيار لتوقف رحلة النمل أو عدد الجولات الكلية للنمل للبحث عن الحل (كل جولة تبدأ من أول عقدة (نثاط) لشبكة المشروع وتتتهي الجولة الواحدة حينما النملات جميعها تصل إلى آخر عقدة (نثاط) لشبكة - المشروع

ق قيمة ثابتة ضمن المدى (0,1) لتحديد مقدار الزيادة في كمية الأثر وبالتالي فهي تحدد نسبة كثافة المادة العطرية التي تعرزها النملة.

(0,1)

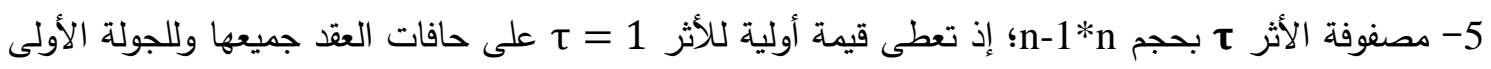

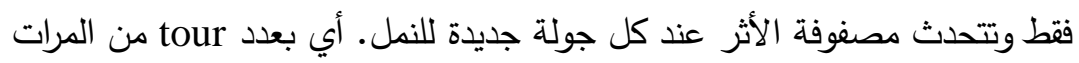

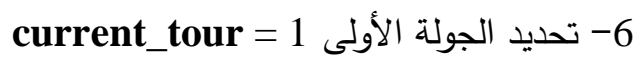


ثانياً/ قراءة ملف شبكة الأعمال:

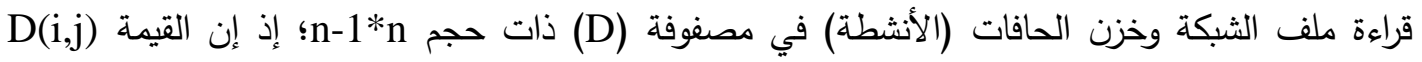
تمثل قيمة النشاط بين العقدة i (الصف) والعقدة j (العمود) وعلى النحو الآتي:

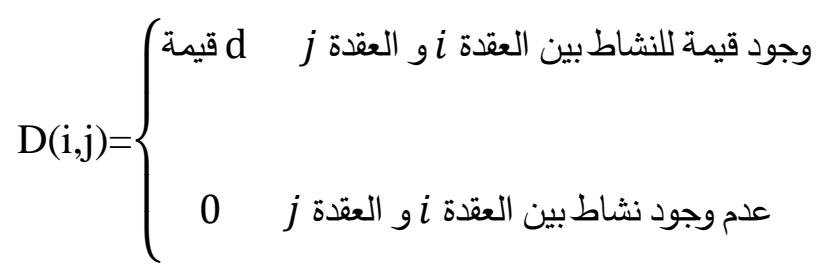

ثالثاً: احتساب الدالة الحسية هذا المعامل يتعلق بكثافة المادة العطرية، وتمثل قدرة النمل في استثعار المادة العطرية. فالدالة الحدسية $\eta_{i j}=\frac{1}{D_{i j}}$ تحسب لمرة واحدة وعلى النحو الآتي:[15,14]. ...equ(2) رابعاً: مرحلة تهيئة النمل لابتداء الجولة: إنشاء مصفوفة المسارات للنملات جميعها tabu m*n فيوضع النمل في العقدة الأولى (بداية المشروع) لتنطلق

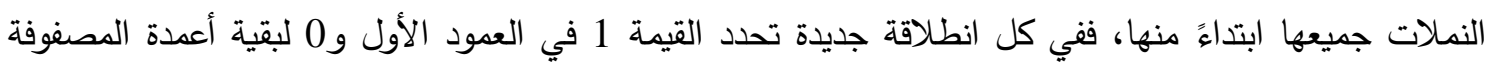
tabu إيذاناً بانطلاقة جديدة للنملات كافة وعدد هذه الانطلاقات بعدد الجولات tour. خامساً: قاعده الانتقال (عملية بناء الحلول): اختيار النملة للعقدة التالية على وفق المعلومات الحسية وتركيز الفيرمون؛ إذ تقوم النملة بالتتقل من العقدة

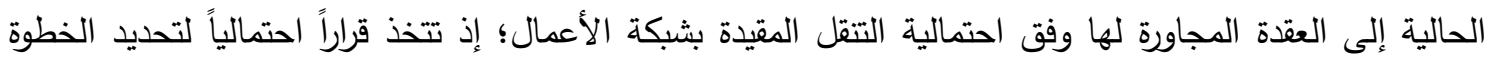

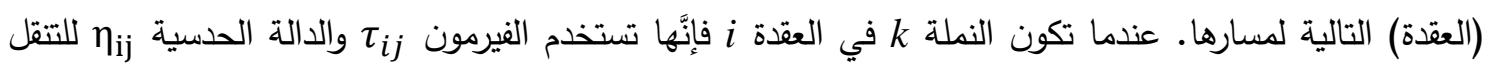

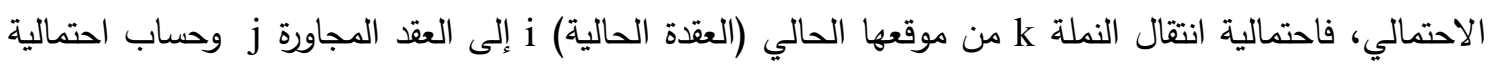
$\mathrm{p}(\mathrm{j})=\tau_{\mathrm{ij}}{ }^{\alpha} * \eta_{\mathrm{ij}}^{\beta}$ اختيار العقدة التالية ز على النحو الآتي: اختيار العقدة التالية تتم بالاعتماد على طريقة عجلة الروليت (roulette - wheel) وعلى النحو الآتي: 1- احتساب احتمالية المسارات:

(4) $\ldots e q u$

$$
\mathrm{p}=\mathrm{p}(\mathrm{j}) / \operatorname{sum}(\mathrm{p})
$$

حيث تمثل p احتمالات انتقال النملة k إلى العقد التالية j. 2- احتساب المجموع التراكمي التلك الاحتمالات:

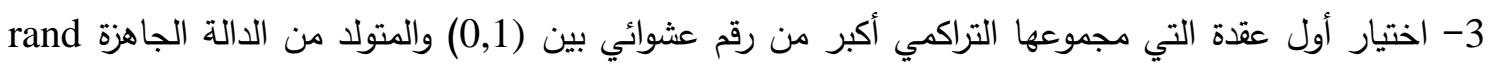

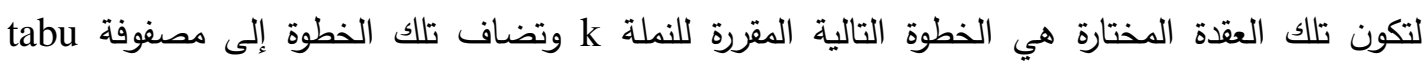


وتستمر النملة بهذا السياق لحين أن تصل إلى هدفها (آخر عقدة بالمشروع) ويطبق هذا على النملات كافة وبالتالي فالمصفوفة tabu ستحوي على مسارات كاملة وللنملات جميعها.

سادساً: احتساب دالة الههف لكل حل (نملة): L(K) for k=1 to m $\mathrm{L}(\mathrm{k})=\sum_{j=1}^{\text {path_length }(k)-1} D(\operatorname{tabu}(k, j), \operatorname{tabu}(k, j+1))$ for $k=1$ to $\mathrm{m}$

.k يمثل طول رحلة النملة path-length (k)

سابعاً: احتساب أفضل رحلة للجولة الحالية current_tour: والمتمثلة بأفضل مسار حرج والاحتفاظ بمساره.

$\mathrm{L}_{\text {best }}$ (current_tour) $=\max (\mathrm{L}(\mathrm{k}))$ for $\mathrm{k}=1$ to $\mathrm{m}$

ثامناً: عملية تحديث المادة العطرية بعد كل جولة: تبدأ بعد أن يكمل النمل كافة جولاتهم (مسارهم) ففي كل مرة تتحرك فيها النملة تترك وراءها بعضاً من المادة العطرية. والهدف من تحديث المادة العطرية هو زيادة الأثر على أفضل المسارات المرتبطة بالحلول الجيدة، وتقليلها على المسارات التي ترتبط بحلول غير جيدة، فالنمل ينجذب (يفضل) نحو المسارات التي تمتلك كمية عالية من المادة العطرية، وعملية تحديث الأثر تتمثل بالآتي: 1 - تصفير مصفوفة نسبة تغير المادة العطرية (الأثر)

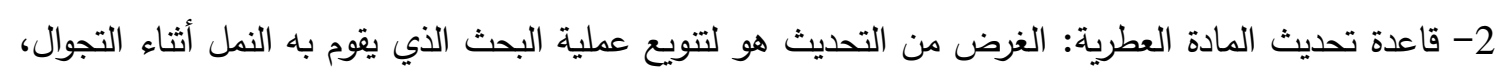

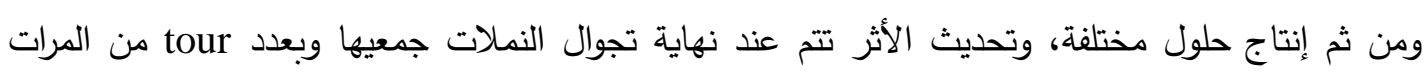
يحدث: - زيادة كمية المادة العطرية: يعزز النمل المادة العطرية بواسطة وضع كمية معينة على الحواف التي يمر بها؛ فبزيادة هذه الكمية ستزداد احتمالية السير على هذا المسار من قبل بقية النمل، إن معادلة تحديث

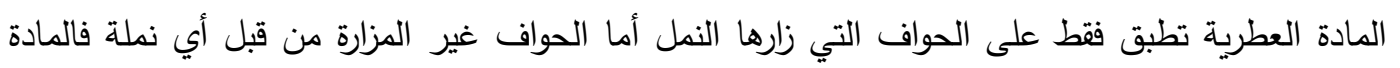
العطرية المترسبة عليها تبقى صفراً. وإنَّ كمية المادة العطرية التي تتركها النملات على العهل كامل مسارها

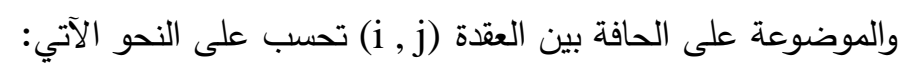

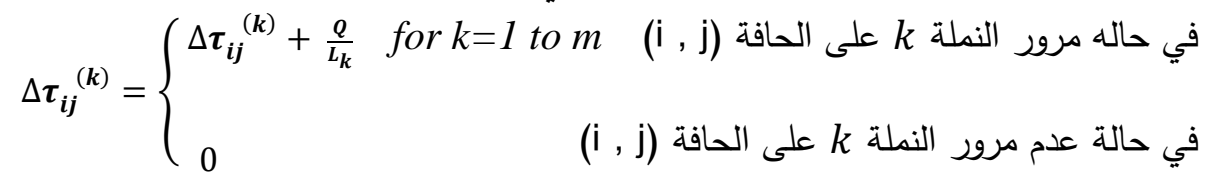
...equ (7)

- - نسبة تبخر (تناقص) للمادة العطرية: ينخفض مستوى (كمية) المادة العطرية التي خلفها النمل وراءه

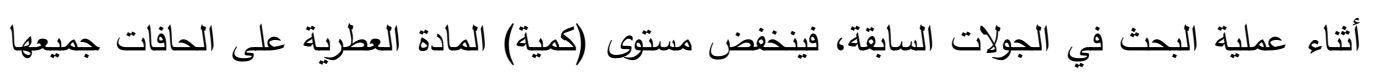
(i,j)

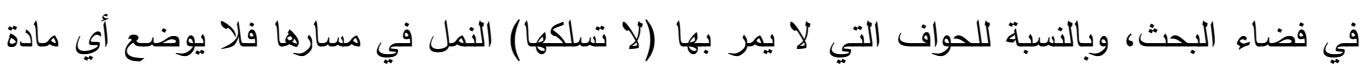

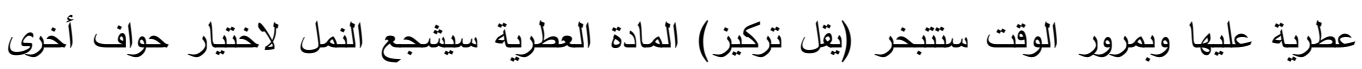
مؤدية بذلك إلى نسيان ذلك المسار . 


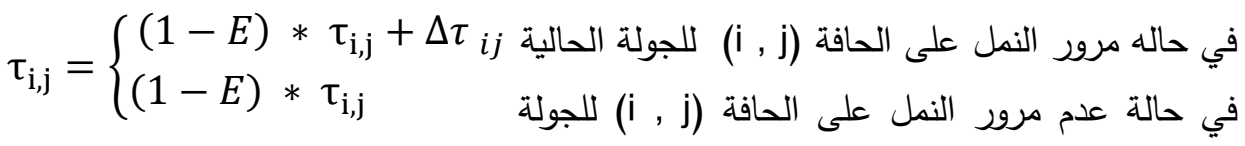

تاسعاً: ابتاء الجولة التالية:

يتم tour > current_tour ففي حالة (عدم اتمام الجولات جميعها) current_tour=current_tour+1

$$
\text { عاشراً: المسار الحرج الأمثل: }
$$

بعد إتمام الجولات جميعها يتم إيجاد الذي يمثل أمثل رحلة (حل) للمسألة. وتبيان المسار الذي حقق الحل الأمثل مع اظهار رسم بياني يوضح الحلول المثلى المحلية عند كل جولة فضلاً عن معدلات الحلول المحلية عند كل جولة.

\section{7- خطوات خوارزمية النمل لثبكة مشروع افتراضية عامة:}

خصصت هذه الفقرة للشرح التفصيلي لخطوات خوارزمية النمل لإيجاد المسار الحرج الأمثل لشبكة أعمال

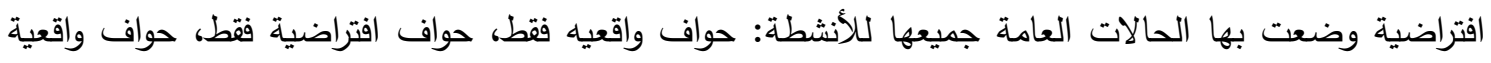
وافتراضية معاً.

1- خطوات خوارزمية النمل لإيجاد المسار الحرج الأمثل: استعين بشبكة افتراضية كما في الثكل (3). كمثال مبسط لتطبيق خوارزمية النمل وعلى النحو الآتي:

2- تهيئة ملف شبكة الاعمال:

تحول الثبكة إلى ملف نوع (dat.) يتضمن السطر الأول عدد العقد التابعة للعقدة الأولى وأسماء تلك العقد وبالسطر التالي قيم الأنثطة التابعة لكل عقدة وهكذا لبقية أسطر الملف؛ إذ إنَّ العقدة الأخيرة التي تمثل نهاية المشروع لا تخزن في الملف، لكونها عقدة ورقية leaf node والجدول (1) يوضح الملف grid6.dat الخاص بالثبكة في الثكل (3).

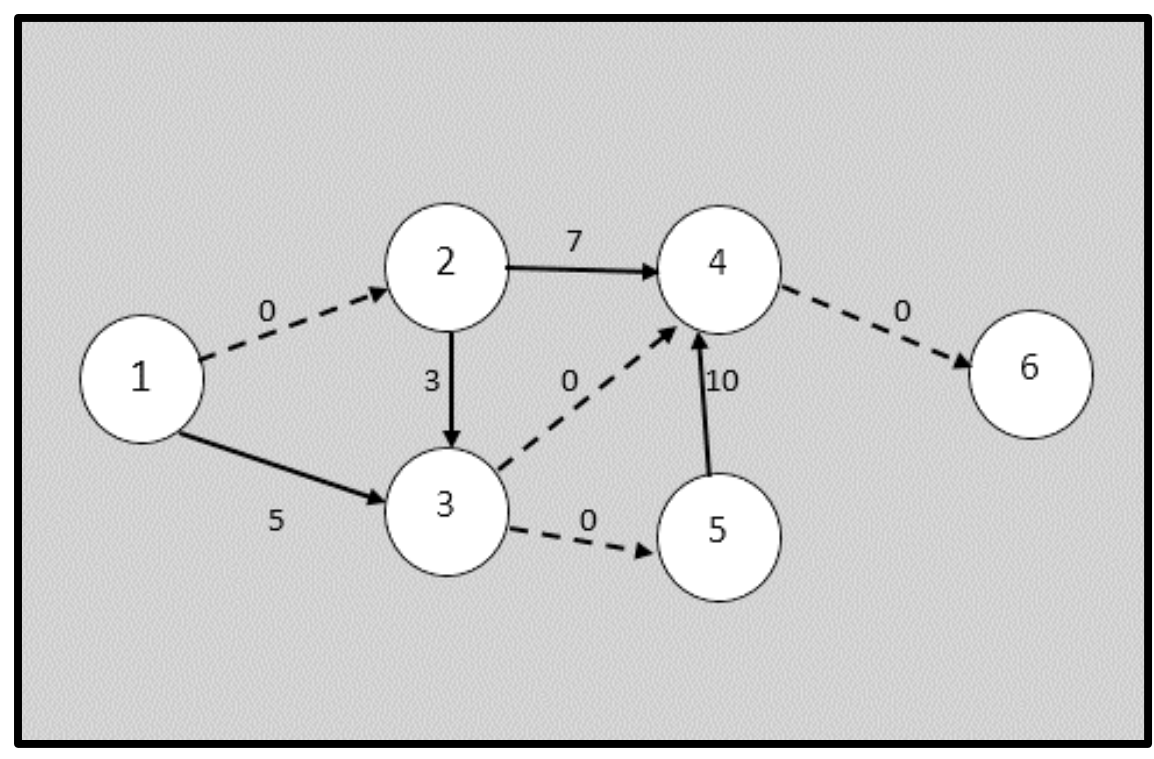

الثكل (3): شبكة إعمال افتراضية. 
الجدول رقم (1): ملف شبكة الأعمال الافتراضية grid6.dat

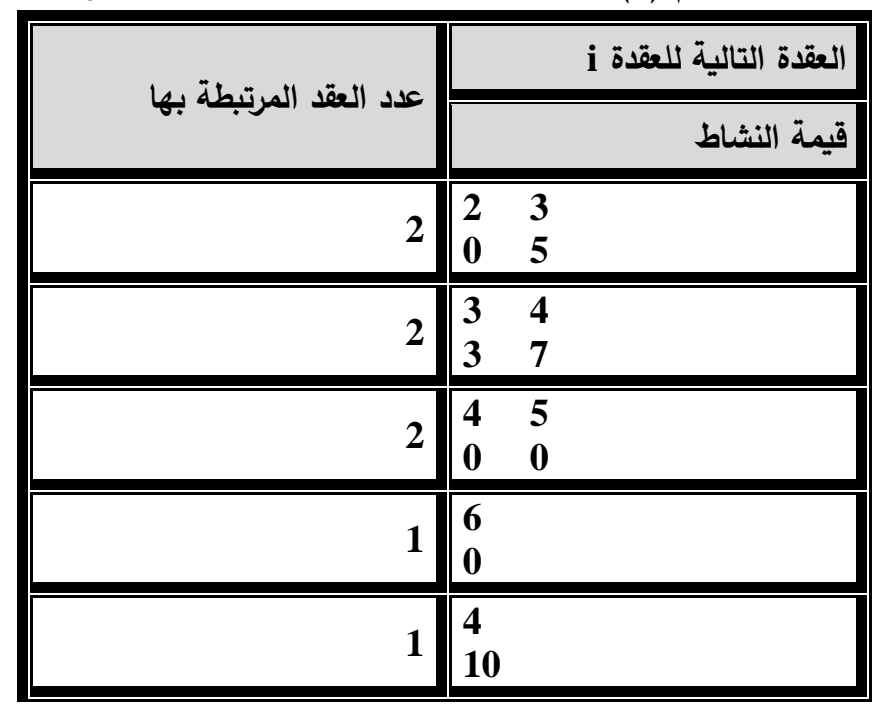

3- قراءة ملف الشبكة الافتراضية (grid6.dat)

يقرأ الملف وتخزن محتوياته في متجه هيكلي array of structure باسم grid وهو متجه يمثل عناصر وكل عنصر يحوي على ثلاثة حقول، هي: no -1 : عدد العقد المرتبطة بالعقدة الحالية .

node -2 : متجه بطول no no مئى أسماء العقد المربوطة بالعقدة الحالية. -3 - مo متجوي على الكلف لتلك الأنشطة، بالنسبة لأنشطة العقد المربوطة بالعقدة الأولى وهكذا $\operatorname{grid}(1) \cdot$ no $=2$

grid (1).nodes $(1:$ no $)=\left[\begin{array}{ll}2 & 3\end{array}\right]$

grid (1). cost $(1:$ no $)=\left[\begin{array}{ll}0 & 5\end{array}\right]$

i المصفوفة (D) توضح الحافات (الأنثطة) بين العقدة i المتمثلة بالصفوف والعقدة j والمتمثلة بالأعمدة،

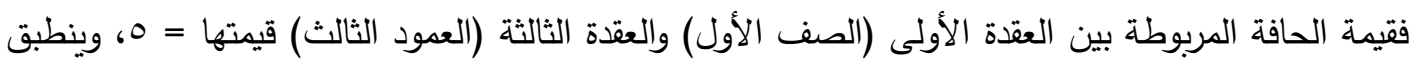
الحال على قيم المصفوفة d جميعها، والقيمة 1- تثير إلى عدم وجود الحافة والقيمة، تثير إلى وجود نشاط وهمي (إذ إنَّ قيمة النشاط الوهمي = صفر ).

$$
\begin{aligned}
& \mathbf{D}(\mathbf{n}-1, \mathbf{n})=\left[\begin{array}{llllll}
-1 & 0 & 5 & -1 & -1 & -1
\end{array}\right. \\
& \begin{array}{llllll}
-1 & -1 & 3 & 7 & -1 & -1
\end{array} \\
& \begin{array}{llllll}
-1 & -1 & -1 & 0 & 0 & -1
\end{array} \\
& \begin{array}{llllll}
-1 & -1 & -1 & -1 & -1 & 0
\end{array} \\
& \left.\begin{array}{llllll}
-1 & -1 & -1 & 10 & -1 & -1
\end{array}\right] 5 * 6 \\
& \text { ii }
\end{aligned}
$$

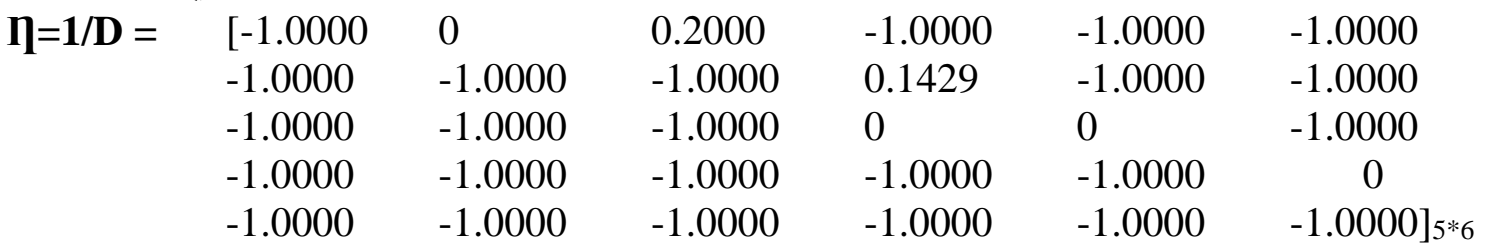


iii المصفوفة عند كل جولة.

iv القيمة الأولية للمادة العطرية المفرزة من قبل النمل في كل جولة. $\Delta \mathrm{T}(\mathrm{n}-1, \mathrm{n})=[0]_{5 * 6}$

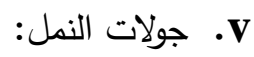

تبتدأ كل جولة بوضع النملات في العقدة الأولى والخطوات التالية تبين تفاصيل بعض الجولات وعلى النحو

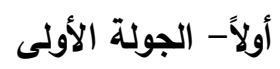

1- توضع النملات الست جميعها على عقدة بداية المشروع كما في مصفوفة Tabu لإيجاد مساراتها لاحقاً.

$\begin{array}{lrlllll}\text { Tabu }(\mathrm{m}, \mathrm{n})= & {[1} & 0 & 0 & 0 & 0 & 0 \\ & 1 & 0 & 0 & 0 & 0 & 0 \\ 1 & 0 & 0 & 0 & 0 & 0 \\ & 1 & 0 & 0 & 0 & 0 & 0 \\ & 1 & 0 & 0 & 0 & 0 & 0]\end{array}$

2- اختيار الخطوة الأولى للنملة الأولى عن طريق الدالة الحدسية الموضحة في المعادلة (3) equ؛ إذ إنَّ العقدة

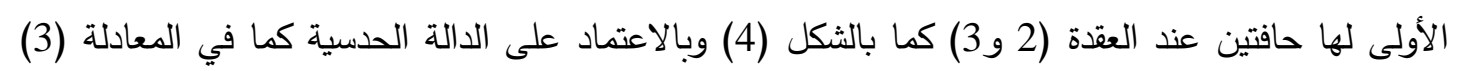

$$
\begin{aligned}
& \mathrm{P}=\left[\begin{array}{ll}
0 & 0.2
\end{array}\right] \\
& \mathrm{P}=\mathrm{P} / \operatorname{sum}(\mathrm{P})=\left[\begin{array}{ll}
0 & 1
\end{array}\right] \\
& \mathrm{Pcum}=\left[\begin{array}{ll}
0 & 1
\end{array}\right] \\
& \text { Select =2 }
\end{aligned}
$$

3- ستتجه النملة الأولى إلى الحافة الثانية لكونها اختيرت عشوائياً والمتمثلة بالعقدة الثالثة، التي قيمة نشاطها= 5

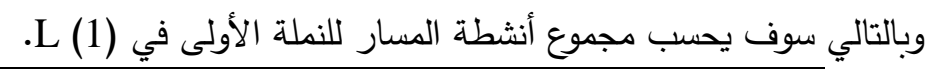

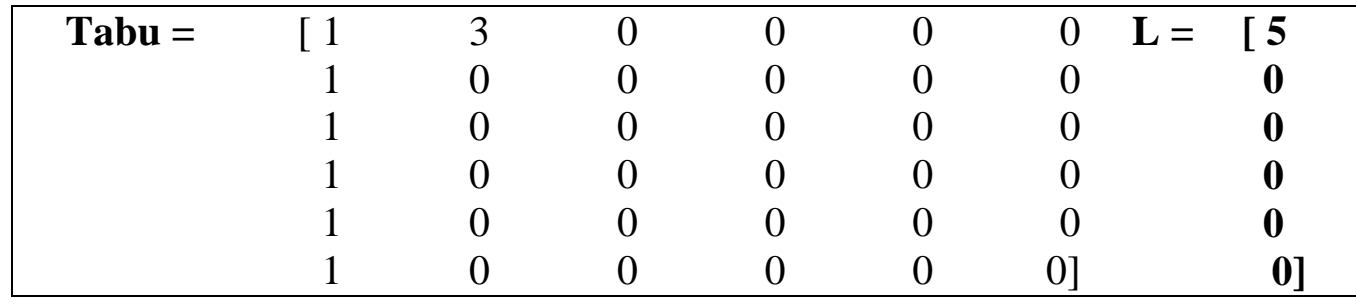

4- وكذلك بالنسبة للخطوة الثانية للنملة الأولى؛ لأن العقدة 3 مربوطة بعقدة 4 و5 بمسارين وهميين وبالتالي يختار أحد هذين المسارين عشوائياً دون اللجوء إلى عجلة الروليت فيتم التحرك إلى العقدة 5.

\begin{tabular}{|c|c|c|c|c|c|c|c|}
\hline \multirow[t]{6}{*}{ Tabu $=$} & {$[1$} & 3 & 5 & 0 & 0 & 0 & $\mathbf{L}=[\mathbf{5}$ \\
\hline & 1 & 0 & 0 & 0 & 0 & 0 & 0 \\
\hline & 1 & 0 & 0 & 0 & 0 & 0 & $\mathbf{0}$ \\
\hline & 1 & 0 & 0 & 0 & 0 & 0 & $\mathbf{0}$ \\
\hline & 1 & 0 & 0 & 0 & 0 & 0 & $\mathbf{0}$ \\
\hline & 1 & 0 & 0 & 0 & 0 & 0 ] & (0] \\
\hline
\end{tabular}
$\mathbf{P}=\left[\begin{array}{ll}0 & 0\end{array}\right]$

Pcum $=\left[\begin{array}{ll}0 & 0\end{array}\right]$

Select $=2$ 
5- بنفس الخطوات السابقة يحدد مسار النملات جميعها باستخدام الدالة الحدسية وعجلة الروليت وحساب مجموع أنشطة مسار كل منها في متجه L وكما مبين أدناه:

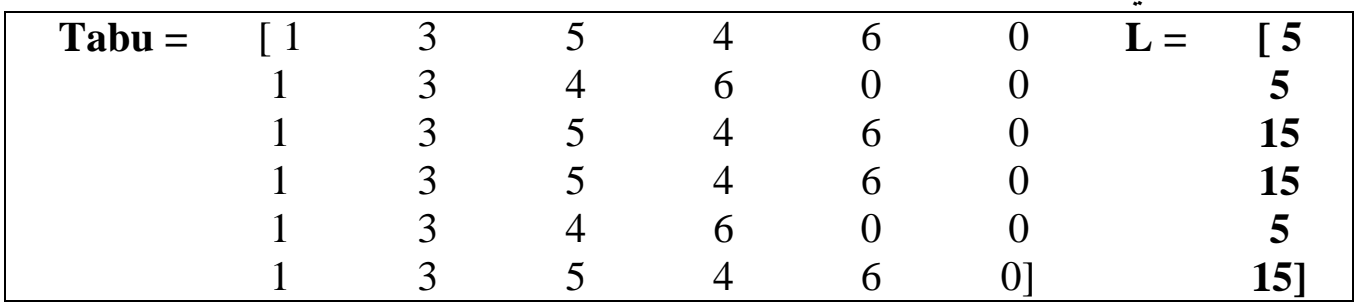

6- في هذه الخطوة نجد أكبر مجموع (L) لمسارات النمل للجولة الحالية ووضعها في 0

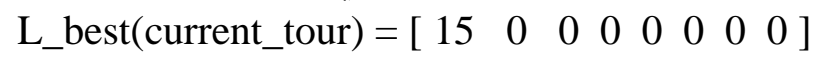

هناك 4 نملات حقتت أكبر مجموع لأنشطة مساراتها والنملات هي [ Pos = فيتم أخذ إحدى

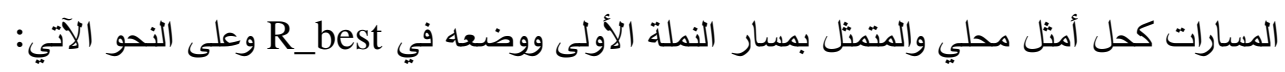
R_best(current_tour) =

$\left.\begin{array}{llllll}{[1} & 3 & 5 & 4 & 6 & 0 \\ 0 & 0 & 0 & 0 & 0 & 0 \\ 0 & 0 & 0 & 0 & 0 & 0 \\ 0 & 0 & 0 & 0 & 0 & 0 \\ 0 & 0 & 0 & 0 & 0 & 0 \\ 0 & 0 & 0 & 0 & 0 & 0 \\ 0 & 0 & 0 & 0 & 0 & 0 \\ 0 & 0 & 0 & 0 & 0 & 0\end{array}\right]$

L_ave $=\left[\begin{array}{lllllllll}11.6667 & 0 & 0 & 0 & 0 & 0 & 0 & 0\end{array}\right]$

7 - تحديد معدل مسارات النمل للجولة الحالية:

8- احتساب المادة العطرية المفرزة $\mathrm{T}$ من قبل النمل في الجولة الأولى:

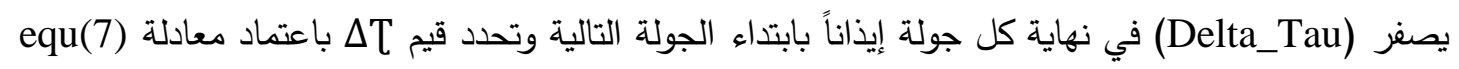

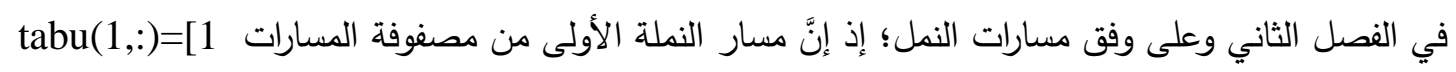

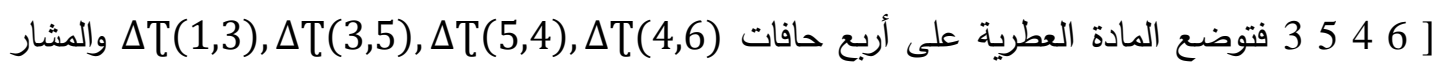

$\Delta \mathrm{T}=\quad \begin{array}{rrrrrr}{[0} & 0 & 0.0267 & 0 & 0 & 0 \\ 0 & 0 & 0 & 0 & 0 & 0 \\ 0 & 0 & 0 & 0 & 0.0267 & 0 \\ 0 & 0 & 0 & 0 & 0 & 0.0267 \\ 0 & 0 & 0 & 0.0267 & 0 & 0]\end{array}$

ثم تضيف النملة الثانية مادة عطرية التي مسارها [ 1 tabu(2,: والمكون من ثلاث حافات هT(1,3), $\Delta \mathrm{T}(3,4), \Delta \mathrm{T}(4,6)$ كل من النملة الأولى والثانية؛ فالقيم الغامقة تمثل كثافة المادة العطرية التي تركتها النملة الثانية على مسارها، والقيم المائلة الغامقة تمثل كثافة تركيز المادة العطرية على الحافة من قبل النملتين الأولى والثانية وعلى النحو تئلئ

$\Delta \mathrm{T}=\quad\left[\begin{array}{rrrrrr}0 & 0 & \mathbf{0 . 1 0 6 7} & 0 & 0 & 0 \\ 0 & 0 & 0 & 0 & 0 & 0 \\ 0 & 0 & 0 & \mathbf{0 . 0 8 0 0} & 0.0267 & 0 \\ 0 & 0 & 0 & 0 & 0 & \mathbf{0 . 1 0 6 7} \\ 0 & 0 & 0 & 0.0267 & 0 & 0]\end{array}\right.$


إذ نلاحظ أنَّ (4T(1,3), $\Delta \mathrm{T}(4,6$ تمثل أعلى تركيز عطري على الحافة بين العقدة (1 و3) والعقدة (4 و6) الموضح في الثكل (3). بسبب كون 6 نملات مرت بهاتين الحافتين، والقيمة 0 تثير إلى عدم وجود مادة لدادئ عطرية ملقاة على تلك الحافات.

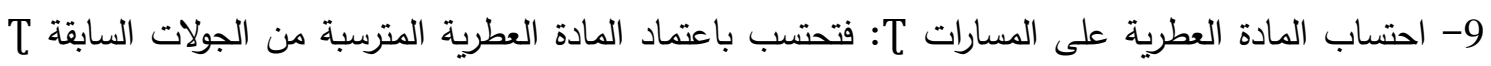

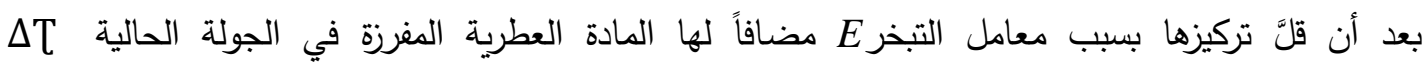

$$
\begin{array}{rrrrrrr}
(\mathrm{n}-1, \mathrm{n})=\mathrm{C} & {[0.9000} & 0.9000 & 1.1667 & 0.9000 & 0.9000 & 0.9000 \\
& & & & & & \\
& 0.9000 & 0.9000 & 0.9000 & 0.9000 & 0.9000 & 0.9000 \\
0.9000 & 0.9000 & 0.9000 & 1.0600 & 1.0067 & 0.9000 \\
0.9000 & 0.9000 & 0.9000 & 0.9000 & 0.9000 & 1.1667 \\
& 0.9000 & 0.9000 & 0.9000 & 1.0067 & 0.9000 & 0.9000] 5 \% 6
\end{array}
$$

\begin{tabular}{|c|c|c|c|c|c|c|c|c|}
\hline \multirow[t]{6}{*}{ Tabu $=$} & {$[1$} & 2 & 3 & 4 & 6 & 0 & $\mathbf{L}=$ & [3 \\
\hline & 1 & 3 & 4 & 6 & 0 & 0 & & 5 \\
\hline & 1 & 2 & 3 & 5 & 4 & 6 & & 13 \\
\hline & 1 & 3 & 5 & 4 & 6 & 0 & & 15 \\
\hline & 1 & 2 & 3 & 4 & 6 & 0 & & 3 \\
\hline & 1 & 3 & 4 & 6 & 0 & $0]$ & & $5]$ \\
\hline
\end{tabular}

وفي نهاية الجولة الأولى زاد التركيز العطري (أكبر من 1) على بعض حافات T في حين أن هناك حافات أخرى حصل لها تبخر فقط والمتمثلة بالقيمة 0.9 وبهذه النتيجة تكون قد انتهت الجولة الأولى التئ.

ثانياً: الجولة الثانية

A . تبدأ الجولة بانطلاق النملات الست جميعها من العقدة الأولى والموضحة مساراتها على النحو الآتي:

B. الاحتفاظ بأمثل حل محلي ومساره ومعدل حلول الجولة الثانية على التوالي: $\mathrm{L}_{-}$best $=\left[\begin{array}{llllllll}15 & 15 & 0 & 0 & 0 & 0 & 0 & 0\end{array}\right]$

$\begin{array}{lllllll}\text { R_best (current_tour) }= & {[1} & 3 & 5 & 4 & 6 & 0 \\ 1 & 3 & 5 & 4 & 6 & 0 \\ 0 & 0 & 0 & 0 & 0 & 0 \\ 0 & 0 & 0 & 0 & 0 & 0 \\ 0 & 0 & 0 & 0 & 0 & 0 \\ 0 & 0 & 0 & 0 & 0 & 0]\end{array}$

L_ave $=\left[\begin{array}{lllllllll}11.6667 & 7.3333 & 0 & 0 & 0 & 0 & 0 & 0\end{array}\right]$

ST الجولة الثانية: C احتساب المادة العطرية المفرزة

$\Delta \mathrm{T}=\quad\left[\begin{array}{rrrrrr}0 & 1.1949 & 0.1333 & 0 & 0 & 0 \\ 0 & 3 & 5 & 4 & 6 & 0 \\ 0 & 0 & 0.1949 & 0 & 0 & 0 \\ 0 & 0 & 0 & 0.2133 & 0.1149 & 0 \\ 0 & 0 & 0 & 0 & 0 & 0.3282 \\ 0 & 0 & 0 & 0.1149 & 0 & 0]\end{array}\right.$


فنلاحظ أنَّ الحافة $\mathrm{LT}(1,2$ بين العقدة 1 والعقدة 2 كانت مسار لثلاث نملات (1,3,5). أمَّا الحافة

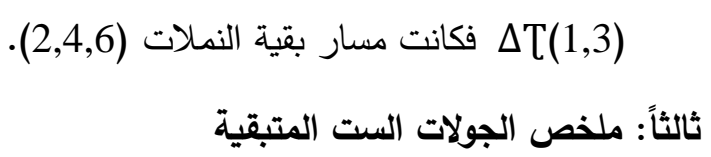

وكما موضح في الجولتين الأولى والثانية سيرفد R_best , L_ave, L_best بالحل المثالي المحلي ومساره ومعدل الحلول على التوالي والجدول (2) يوضح القيم النهائية لها بعد انتهاء الجولات الثمانِ. الجدول (2): ملخص لنتائج الجولات الثمان لمسألة الثبكة الافتراضية.

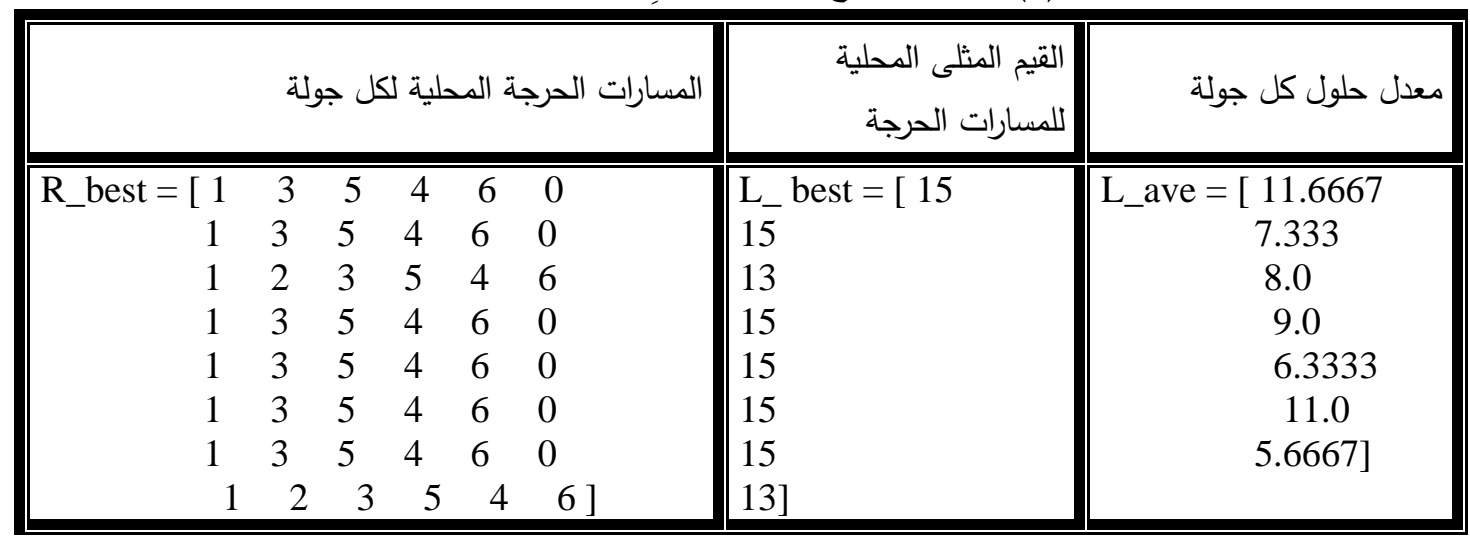
$\mathrm{T}=\left[\begin{array}{llllll}0.4305 & 1.8394 & 1.3918 & 0.4305 & 0.4305 & 0.4305\end{array}\right.$

$\begin{array}{llllll}0.4305 & 0.4305 & 1.6640 & 0.6059 & 0.4305 & 0.4305 \\ 0.4305 & 0.4305 & 0.4305 & 2.2320 & 0.8238 & 0.4305 \\ 0.4305 & 0.4305 & 0.4305 & 0.4305 & 0.4305 & 2.8008 \\ 0.4305 & 0.4305 & 0.4305 & 0.8238 & 0.4305 & 0.4305]\end{array}$

رابعاً: النتائج النهائية لمسألة الشبكة الافتراضية كما موضح في الثكل (4) للواجهة الرئيسة لبرنامج ANTOGPN حددت 6 نملات و8 جولات وغيرها من المعاملات (على يسار النافذة) وبالنقر على زر Optimal Solution يتضح أنَّ أمثل مسار حرج بطول 5 حافات [

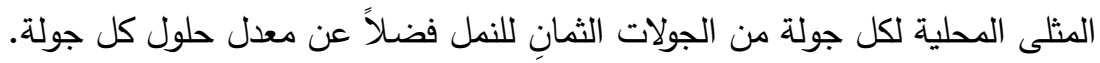

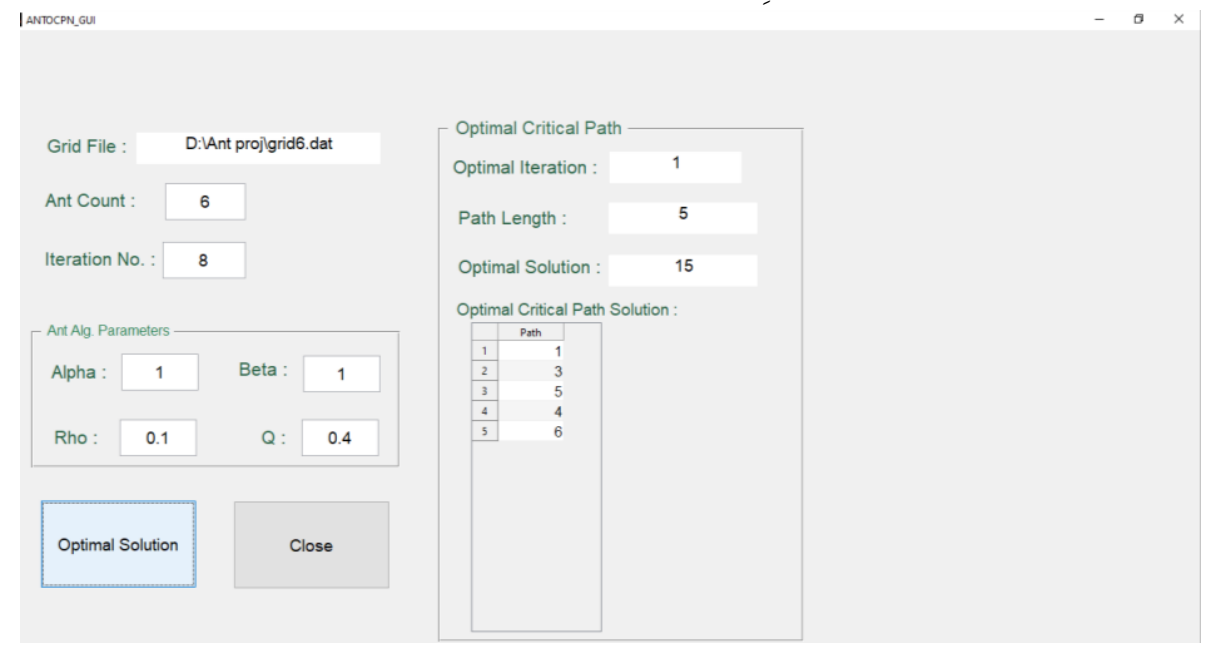

الثكل (4): واجهة برنامج ANTOGPN بعد الحصول على أمثل حل للمسألة. 


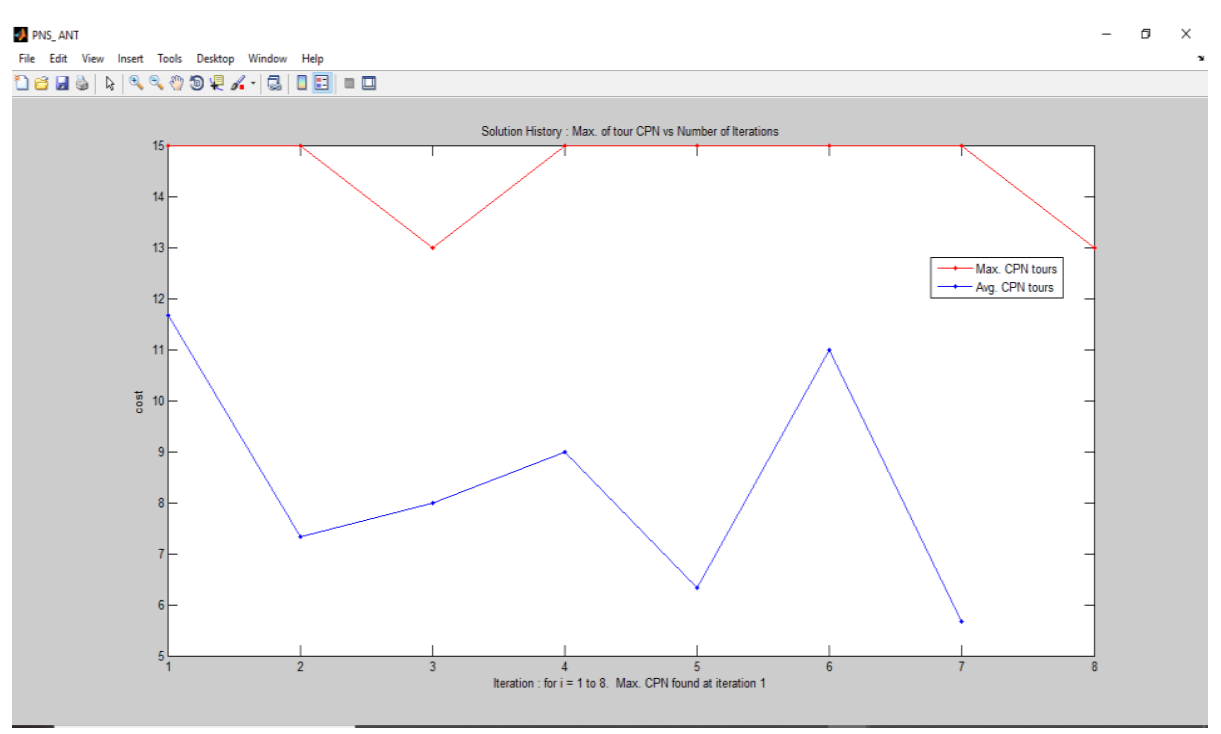

الثكل (5): مخطط بياني يوضح الحلول المثلى ومعدلاتها لثمانِ جولات.

8- الاستنتاجات:

i.. بالإمكان التعامل مع عقد مربوطة بنشاط وهمي واحد أو أكثر ، وعقد مربوطة بنشاط حقيقي واحد أو أكثر ، وعقد مربوطة بواحدة أو أكثر من الأنشطة الوهمية والحقيقية معاً.

ii. بتحليل النتائج التجريبية لبرنامج ANTOCPN الذي اعتمد على التئ عجلة الروليت والدالة العشوائية الجاهزة

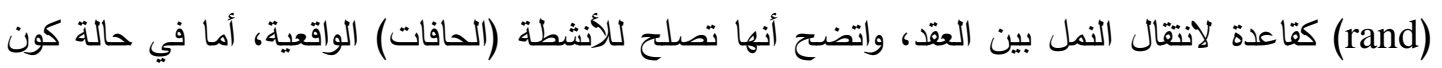
أحد الحافات أو جميعها وهمية (قيمتها =0) ستفشل عجلة الروليت في اختيار تلك الحواف (فرصة اختيارها

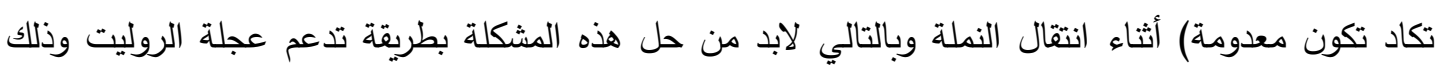
باعتماد الدالة العشوائية (randi) لإعطاء فرص (احتمالات) متساوية لاختيار الحواف بنوعيها (الواقعية

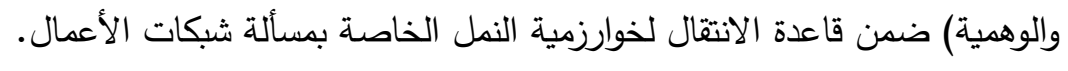

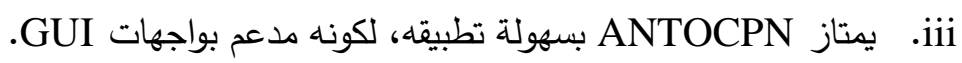
iv v. أسفرت نتائج تطبيق ANTOCPN بالحصول على أمثل حل للمسار الحرج الذي تحققه مسارات عدة، مما يعطي حرية لمدير المشروع (متخذ القرار) باتخاذ القرار الأنسب في اختيار إحداها وعلى وفق متطلبات بيئة

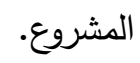
vi. التقانات الذكائية لإيجاد المسار الحرج الأمثل لشبكة المشاريع تعمل على نحو مختلف ومستقل عن نظيرتها من الطرائق التقليدية (كبحوث العمليات) لإيجاد المسار الحرج الأمثل. لمان.

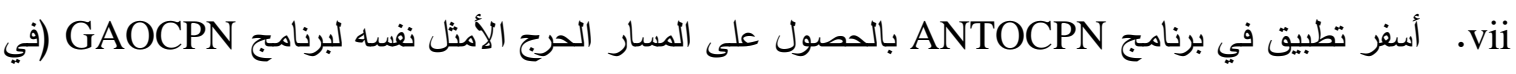
دراسة سابقة) لكن بعدد خطوات أقل وبالتالي فإن ANTOCPN هو أسرع بالوصول على إلى الحل الأمثل من 


\section{المصادر}

[1] Youmei Li, Zongben Xu1, (2003) " An Ant Colony Optimization Heuristic for Solving Maximum Independent Set Problems" Proceedings of the Fifth International Conference on Computational Intelligence and Multimedia Applications (ICCIMA’03). 0-7695-1957-1/03 IEEE.

[2] Zhao, T. and Tseng, C-L, (2003), "A note on activity flouts in activity-onarrow networks", Journal of the Operational Research Society, Vol. (54), PP.1296 -1299.

[3] Blum, C., and Dorigo, M. (2004), "The hyper-cube framework for Ant Colony

Optimization" Transactions on Systems, Man and Cybernetics Part B: Cybernetics, Vol. 34 Issus 2.

[4] Dorigo, M. and Gambardella, L.M., (1997), "Ant colony system: a cooperative learning approach to the traveling salesman problem", IEEE Transactions, Evolutionary Computation, Vol. (1), PP. 53-66.

$$
\begin{aligned}
& \text { الحكيم، لطيف عبد رجب والمنصوري، عبد الجليل آدم، (1987)، "مدخل الى بحوث العمليات"، دار } \\
& \text { دمشق، دمشق، سوريا. } \\
& \text { الثمرتي، حامد سعد نور والزبيدي، علي خليل، (2007)، "مدخل الى بحوث العمليات"، دار مجدلاوي } \\
& \text { للنشر والتوزيع، عمان، الأردن. } \\
& \text { نجم، نجيب عبد المجيد، (2012)، "استراتيجية استخدام أساليب جدولة المشروع، بيرت والمسار الحرج }
\end{aligned}
$$

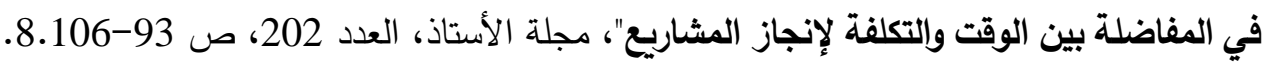

$$
\begin{aligned}
& \text { العبيدي، أسيل إسماعيل علي، د. مهى عبد الرحمن حسّو،(2011) تقنية مستعمرة نمل مقترحة لتشخيص } \\
& \text { الأورام في الصور الطبية. جامعة الموصل، كلية علوم الحاسوب والرياضيات. }
\end{aligned}
$$

[9] J. Jaya , K. Thanushkodi, (2010) "Segmentation of MR Brain Tumor using Parallel ACO" International Journal of Computer and Network Security, Vol 2, No. 6, June 2010. IEEE.

[10] Dorigo, M. and Stützle, Thomas, (2004), "Ant Colony Optimization", MIT Press, London, England.

[11] Critical Path Method in a Project Network using Ant Colony Optimization,(N. Ravi Shankar, P. Phani Bushan Rao, S. Siresha and K. Usha Madhuri Department of Applied Mathematics, GIS, GITAM University, Visakhapatnam, INDIA) 2011.

[12] Designing a genetic algorithm to find the optimum critical solution for the enterprise business network. 
تصميم خوارزمية جينية لإيجاد المسار الحرج الأمثل لشبكة أعمال المشاريع(GAOCPN)، (د. سماء

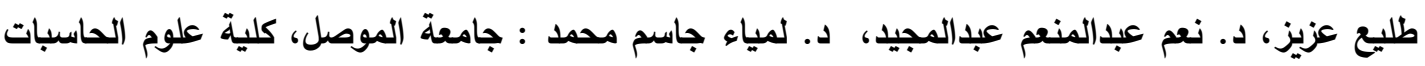

والرياضيات ، 2011).

[13] The use of the ant colony algorithm to find the optimal business scheduling solution

(د. خالد ضاري الطائي (اشراف)، الباحث / ابتهال هاشم رحيم، كلية المنصور الجامعة، الجامعة المستصرية

[14] Abdullah, Hazem, et. Al., (2009), "Using Ant Colony Optimization algorithm for solving project management problems", Expert Systems with Applications, Vol. (36), PP. 10004-10015.

[15] Sim, K. M. and Sun, W. H. (2003),"Ant Colony Optimization for routing and load balancing: Survey and new directions", IEEE Transactions on Systems, Man, and Cybernetics - Part A: Systems and Humans, Vol. 33 Issus 5. 